\title{
CONSTRUCCIÓN DE NORMAS: UNA EXPERIENCIA DESDE EL CLIMA DE AULA*
}

\section{CONSTRUCTION OF RULES: AN EXPERIENCE FROM THE CLASSROOM ENVIRONMEN}

\section{Anderson Geovany Rodríguez-Buitrago**}

\section{Resumen}

Objetivo. Este documento presenta algunos análisis sobre la percepción que tienen los estudiantes respecto a la construcción de normas dentro del clima de aula. Metodología. Se analizaron datos derivados del macroproyecto "Instituciones Educativas Vivas", a través de encuestas realizadas a estudiantes de la Institución Educativa Técnica Internado San Francisco de Loretoyaco de Puerto Nariño (Amazonas). La investigación se llevó a cabo bajo la metodología de tipo no experimental, descriptiva. Resultados. Se pudo constatar la importancia de generar una cultura de participación. Conclusión. Entre las principales conclusiones se pudo evidenciar la importancia del docente en la construcción participativa de normas.

Palabras clave: construcción de normas, clima de aula, clima escolar, estudiantes.

\section{Abstract}

Objective. This paper presents some analyses of the student's perception of the construction of rules within the classroom environment. Methodology. Data derived from the macro-project "Living Educational Institutions" was analyzed through surveys applied to students of the Educational Institution San Francisco de Loretoyaco. The research was carried out under the non-experimental, descriptive methodology. Results. It was possible to confirm the importance of generating a culture of participation. Conclusions. Among the main conclusions, it was possible to demonstrate the importance of the teacher in the participatory construction of rules.

Key words: rules construction, classroom environment, school environment, students.

\footnotetext{
* La presente investigación forma parte del macroproyecto "Instituciones Educativas Vivas" de la Facultad de Ciencias de la Educación y Humanidades de la Fundación Universitaria Juan de Castellanos, específicamente de la línea clima escolar.

${ }^{* *}$ Fundación Universitaria Juan de Castellanos. Boyacá, Colombia. E-mail: arodriguez@jdc.edu.co -

(D) orcid.org/0000-0002-8609-7813. Google Scholar
} 
Construcción de normas: una experiencia desde el clima de aula

\section{Introducción}

Uno de los aspectos fundamentales que se abordan en la dinámica del clima de aula es la construcción participativa de normas. Es necesario reflexionar sobre la importancia que tiene la participación en el afianzamiento de las normas dentro del contexto escolar. Como lo plantea Sánchez (2009), "las normas son un recurso educativo para facilitar la convivencia y lograr metas, no previenen de manera absoluta los conflictos, pero sí pueden ayudar a aliviarlos" (p. 323).

Entre los errores que se pueden cometer en el contexto de aula, encontramos que en ocasiones se hacen actividades muy mecánicas y no se aprovechan los marcos de autonomía. Los proyectos y las normas a veces se convierten en declaraciones de intenciones que ni los docentes y estudiantes conocen. También es verdad, decimos y afirmamos, que es necesaria la participación de los implicados, pero pocas veces se propende por una participación real, una participación que permita decidir aspectos fundamentales de convivencia, del desarrollo educativo y de las prácticas escolares. Eso también afecta los mecanismos de gobierno y la cultura democrática de las instituciones educativas que pueden ser más administrativistas, más centradas en las normas construidas de manera externa, que en las personas y la cultura de la participación.

Nos enfrentamos a la necesidad de introducir mecanismos de cambio, ya que la adecuada construcción de normas de comportamiento y su influencia en el clima de aula requiere de un involucramiento de docentes y estudiantes a partir de sus propias realidades. En consonancia, para involucrarse en una norma hace falta conocerla, es necesario analizarla, preguntarla y comprenderla. Como lo menciona Sánchez (2009), "el objetivo es potenciar la conciencia de grupo y lograr el compromiso de asimilar conocimientos, afianzar destrezas y valores, y conseguir la cooperación de todos los miembros en la consecuencia de tales fines” (p. 324).

Es importante entender que la efectividad del cambio en el contexto escolar no viene de las grandes ideas aisladas, sino por el esfuerzo conjunto y continuado basado en la autonomía. Es necesario incluir el trabajo colaborativo y participativo en las aulas, introducir pequeños cambios. El cambio no es una situación puntual, sino que es una meta a la que nos podemos aproximar, donde docentes y estudiantes sean los actores principales del proceso.

La relación entre estos actores se da especialmente en el clima del aula, por tanto, hablar de clima de aula permite reflexionar en torno a las vivencias y experiencias que se dan en el contexto dentro del cual transcurre gran parte del día a día de docentes y estudiantes. Respecto al clima de aula, Martínez (1996) realiza una importante definición: 
(...) definimos pues el clima o ambiente de aula como una cualidad relativamente duradera, no directamente observable, que puede ser aprendida y descrita en términos de las percepciones que los agentes educativos del aula van obteniendo continua y consistentemente sobre dimensiones relevantes de la misma como son sus características físicas, los procesos de relación socioafectiva e instructiva entre iguales y entre estudiantes y profesor, el tipo de trabajo instructivo y las reglas, y normas, que lo regulan. (p. 118)

Como vemos en la definición anterior, la construcción de normas se encuentra interrelacionada con lo que pasa en el contexto de aula. Se hace pues necesario que dentro del clima de aula se generen espacios para que el estudiante contribuya, por medio de acuerdos, al funcionamiento de la clase, con el fin de que se genere respeto por las diferencias y el desarrollo de la tolerancia, el desarrollo de habilidades sociales, la escucha activa, la empatía, la autorregulación de las emociones, el cuidado, las buenas palabras y el enriquecimiento de la relaciones interpersonales.

Estos aspectos pueden llevar a una revisión de las prácticas autoritarias que en ocasiones emergen en la relación docente-estudiante. Normas y sanciones deben pasar por un consenso, tenemos que usar más el diálogo y construir las cosas juntos. El clima de aula y el clima escolar en el colegio pueden tener un proceso de justicia restaurativa que posibilite convertir estas experiencias en un proceso de formación y enseñanza dentro del contexto educativo. Esto genera impacto no solo en los procesos relacionados con la convivencia armónica y pacífica sino también en la motivación por aprender, es decir, que la construcción participativa de normas posibilita el compartir significados para el desarrollo personal y el grado al que se pertenece, en el caso del estudiante, o del grado a cargo, en el caso del docente. Al respecto, Gómez, Mir y Serrat (2007) mencionan que "estamos convencidos de que el aprendizaje mejora ostensiblemente cuando el grupo-clase está cohesionado, los alumnos se conocen, existe un clima afectivo positivo y hay una buena relación interpersonal” (p. 208).

Ahora bien, dentro de la relación entre la construcción de normas y el clima de aula es necesario tener presente que los conflictos, se quiera o no, van entrando al aula y a la Institución Educativa. Podemos percibir que nuestras ideas e intereses son diferentes, pero no necesariamente incompatibles, es decir, podemos pensar que los conflictos son negativos, pero estos se pueden convertir en un hecho educativo a partir de la reflexión sobre las normas construidas participativamente. Qué importante que los conflictos se vean como oportunidades para crecer, adquirir competencias y desarrollarnos. Estos pueden constituirse en oportunidades para el aprendizaje de habilidades prosociales que posibiliten la convivencia armónica y pacífica. 
Esta relación y construcción de normas requiere del espíritu gestor para consolidar una comunidad de investigación, como lo expresa Lipman (1992):

\begin{abstract}
(...) la construcción de una comunidad de investigación es un logro más substancial que la mera idea de una ambiente abierto. Hay ciertas actitudes que son requisitos previos: la disponibilidad hacia la razón, el respeto mutuo (de los niños entre sí y de los niños y profesores entre sí) y una ausencia de adoctrinamiento. (p. 118)
\end{abstract}

Por tanto, esta reflexión basada en la investigación se ha convertido en punto de partida en la Institución Educativa Técnica Internado San Francisco de Loretoyaco de Puerto Nariño (Amazonas). Desde su fundación, la Institución se ha dedicado a formar líderes comunitarios orientados a gestar empresas agropecuarias y turísticas. Para ello vincula docentes en su planta, con perfil para el desempeño dentro de cada área obligatoria. En lo que respecta a la Básica Secundaria y la Media Vocacional, la mayoría de los educadores provienen de diferentes partes del país, como son los departamentos de Boyacá, Chocó, Santander, Cundinamarca, Risaralda y La Guajira. La Institución está localizada en la zona rural del Resguardo Aticoya, a un kilómetro del casco urbano de Puerto Nariño. El municipio está conformado por el casco urbano y 21 comunidades que están localizadas a orillas del río Amazonas y del río Loretoyaco ${ }^{1}$.

La mayoría de la población estudiantil pertenece a las etnias presentes en la región, siendo la más predominante los Ticuna, seguida por los Yaguas, Cocamas, Mestizos y los Colonos en minoría. Los estudiantes ticunas son personas amables, colaboradoras, respetuosas e inteligentes. Para el trabajo, utilizan la ayuda mutua, conocida como Mingas. Les encanta aprender hábitos y costumbres buenas y "malas" de otras culturas, pero siempre conservan su identidad cultural. La actividad económica está fundamentada en suplir las necesidades básicas, produciendo únicamente para el hogar. Por otro lado, las artesanías se elaboran para el comercio y los ingresos generalmente son utilizados para el bienestar doméstico ${ }^{2}$.

La construcción de normas de manera participativa y su relación con el clima de aula se convirtieron en tema de interés para los integrantes de la comunidad educativa, teniendo presente la diversidad cultural de la Institución ya que, como lo expresa Besalú (2002), "no se trata de inventar nada, sino de recrear la mejor tradición pedagógica, aquella que siempre ha tenido claro que para educar a las personas hay que conocerlas, respetarlas y acogerlas en su diversidad" (p. 242). Por tanto, la apertura a lo ajeno, a lo distinto en contextos de lo tradicional, conlleva en sí misma un reto, el cual es la complementariedad que se da no solo desde lo oral sino también en lo escrito, por cuanto permite a partir de la palabra y la escritura dar a conocer la riqueza de las culturas y la unión que puede darse entre los

\footnotetext{
${ }^{1}$ Tomado de las conversaciones con la hermana Sor Edelmira Pinto, coordinadora de la Institución.

${ }^{2}$ Tomado de las conversaciones con la hermana Sor Edelmira Pinto, coordinadora de la Institución.
} 
integrantes de la comunidad educativa. Esto implica comprender que la educación tanto ínter como multicultural deberían generar competencias básicas de atención a la diversidad de manera incluyente y participativa.

A partir de lo expuesto anteriormente, se formuló como pregunta de investigación: ¿De qué manera determinar la relación entre la construcción de normas y el clima de aula? Desde esta formulación, se estableció como objetivo determinar la relación entre la construcción de normas y el clima de aula.

En esta contribución se presenta, desde la percepción de los estudiantes de Básica Secundaria, la importancia de la construcción de normas, especialmente en lo relacionado a la claridad de las normas y la participación en la elaboración de las mismas y el clima de aula. Se aportan elementos de análisis a los contextos de aula e institucionales desde una mirada a las representaciones que tienen los estudiantes sobre su rol activo en las normas de aula, teniendo presente que a la escuela se va a pensar y comprender, y desde allí se construye el pensamiento y las competencias tanto para la vida personal como para la vida en sociedad.

\section{Metodología}

La presente investigación forma parte del macroproyecto "Instituciones Educativas Vivas" de la Facultad de Ciencias de la Educación y Humanidades de la Fundación Universitaria Juan de Castellanos, específicamente de la línea Clima Escolar.

La investigación se fundamentó en el paradigma mixto (cuantitativo y cualitativo) de tipo no experimental-descriptiva, pues se realizó sin manipular deliberadamente las variables, pero sí se realizó una interpretación y análisis de los datos obtenidos. Como lo señala Sampieri (1997): "Lo que hacemos en la investigación no experimental es observar fenómenos tal y como se dan en su contexto natural, para después analizarlos" (p. 245). Es decir, se observó la construcción de normas de clase desde la percepción que tienen los estudiantes respecto a la claridad de las normas, la participación en la elaboración de las mismas y el clima de aula. Se observó el fenómeno tal como se presenta en su contexto natural, para después ser analizado.

La investigación se desarrolló en la Institución Educativa Técnica Internado San Francisco de Loretoyaco, ubicada en el municipio de Puerto Nariño (Amazonas). Es una Institución que se caracteriza por el mejoramiento continuo de sus procesos y la satisfacción de las necesidades de sus estudiantes y padres de familia y/o acudientes. La población objeto de estudio estuvo conformada por estudiantes de Educación Básica Secundaria y Media con edades entre los 11 y los 17 años, de los cuales se seleccionó una muestra de 27 (13 niñas y 14 niños). La técnica utilizada para la recolección de la información fue la encuesta estructurada denominada: Clima Escolar “Ambientes socioeducativos”, versión 4. Esta fue proporcionada por el Centro 
Construcción de normas: una experiencia desde el clima de aula

de Investigación de la Facultad de Educación y Humanidades de la Fundación Universitaria Juan de Castellanos.

El procedimiento para la recolección de información fue el siguiente: se reunió a los niños en las aulas de la Institución, previa revisión de los consentimientos firmados por los padres de familia, y se les explicó el objetivo de la encuesta y de la investigación, y se resolvieron algunas dudas. A continuación, se procedió a entregarles el formulario de encuesta, y en la medida en que se avanzó se aclararon inquietudes de forma individual. Culminado el proceso de aplicación de las encuestas se procedió a revisarlas y establecer cuáles estaban incompletas o ilegibles, y posteriormente se llamó a cada niño para culminar el proceso de diligenciamiento.

\section{Resultados}

Los resultados son derivados de las categorías de análisis e indicadores identificados para establecer la relación entre la construcción de normas de clase y el clima de aula, a saber (Tabla 1):

Tabla 1. Dimensiones y categorías de análisis: Construcción de normas y clima de aula

\begin{tabular}{|c|c|c|}
\hline Dimensiones & Categorías de análisis & Indicadores \\
\hline \multirow{2}{*}{ Construcción de normas } & Claridad de las normas & $\begin{array}{l}\text { Desde el comienzo del año escolar el } \\
\text { profesor explicó la buena conducta } \\
\text { en el aula. } \\
\text { Las normas de clase son claras para } \\
\text { los estudiantes. }\end{array}$ \\
\hline & $\begin{array}{l}\text { Participación en la elaboración } \\
\text { de las normas }\end{array}$ & $\begin{array}{l}\text { El profesor pide la opinión de los } \\
\text { estudiantes sobre el funcionamiento } \\
\text { de la clase. } \\
\text { Los estudiantes participan en la } \\
\text { selección de las normas de clase. }\end{array}$ \\
\hline Clima de aula & $\begin{array}{l}\text { Desarrollo de las actividades } \\
\text { dentro del aula }\end{array}$ & $\begin{array}{l}\text { En el curso, siempre toma algo de } \\
\text { tiempo el inicio de las actividades } \\
\text { programadas mientras se hace } \\
\text { silencio. } \\
\text { Se pierde mucho tiempo porque los } \\
\text { estudiantes interrumpen la clase. }\end{array}$ \\
\hline
\end{tabular}

Fuente: elaboración propia. 


\section{Construcción de normas de clase}

Es común que hoy en día se intente normativizar todo, pero es necesario tener presente que la calidad de las normas reside en la autonomía propia de las personas para que ellas puedan desarrollar sus ideas y aportar de manera democrática y participativa. Autonomía, no independencia, es decir, darles márgenes a los actores educativos para que trabajen en equipo y de manera proactiva. Como lo plantean Bolívar y Balaguer (como se citó en Ministerio de Educación Nacional, 2014):

(...) formación para el ejercicio de la ciudadanía, como proceso pedagógico, demanda enseñar las competencias necesarias para consolidar una comunidad democrática, y estructurar los procesos educativos con acciones que permitan la participación activa en la resolución de problemas cotidianos, la construcción de las normas y la resolución pacífica de los conflictos. (p. 14)

Por tanto, se aborda la construcción de normas como dimensión a través de las siguientes categorías: claridad de las normas y participación en la elaboración de las normas.

\section{Claridad de las normas}

En este aspecto, se tiene presente no solo si las normas son socializadas y explicadas por parte de los docentes al comienzo del año escolar, sino además el conocimiento y claridad de las mismas por parte de los estudiantes (ver Figura 1):

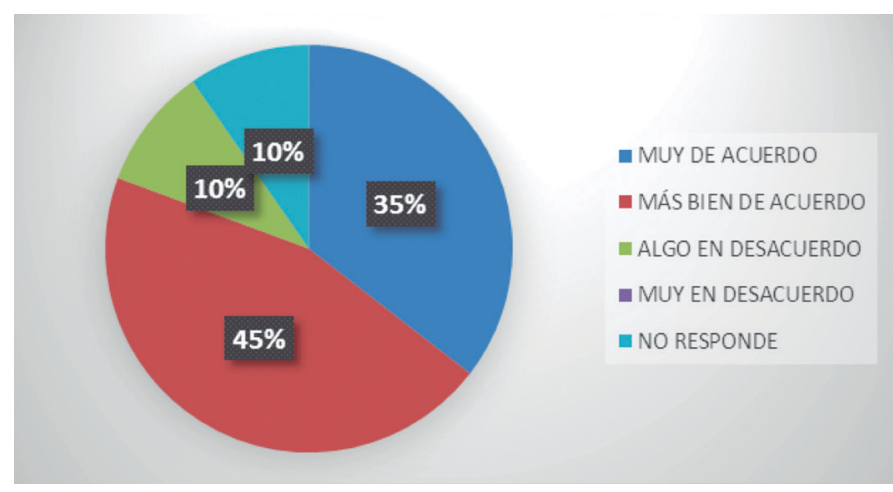

Fuente: Encuesta aplicada a estudiantes de la Institución Educativa Técnica Internado San Francisco de Loretoyaco (Puerto Nariño, Amazonas).

Figura 1. Desde el comienzo del año escolar, el profesor explicó la buena conducta en el aula. 
Construcción de normas: una experiencia desde el clima de aula

Se evidencia que el 45\% de los estudiantes está más bien de acuerdo en que al comienzo del año escolar el docente explicó la importancia y necesidad de la buena conducta en el aula. Un 35\% se muestra muy de acuerdo, frente al 10\% que considera estar algo en desacuerdo y el $10 \%$ que no responde.

La explicación de la buena conducta en el aula se convierte en tema de interés, el centro no son las normas sino las personas que las vivencian e interiorizan. El énfasis debe estar en la mejora permanente de las dinámicas relacionadas con la convivencia, ya que la falta de explicación y comprensión puede desencadenar en conflictos de manera permanente. Generar una cultura de diálogo y respeto entre los integrantes de la comunidad educativa es uno de los principios rectores dentro del clima aula. Para esto, es necesario que tanto docentes como estudiantes internalicen las normas a partir de su explicación, así podrán aportar elementos para mejorar su capacidad de conectarse y empatizar con otros, ya que emoción y razón no se pueden separar.

Somos productores de condiciones, podemos tomar el control de las situaciones y emprender la creatividad para las soluciones a los problemas. Esto tiene que ver con la proactividad del profesorado, viéndola como el arte de mejorar el futuro manejando el presente a través de la socialización y explicación sobre la importancia de la buena conducta. Igualmente, es importante que las normas sean claras para los estudiantes (ver Figura 2).

El estilo de educador dialogante, participativo y que fomenta la convivencia, es claramente más productivo en los resultados obtenidos con sus alumnos, pues contribuye a que se involucren en el proceso de enseñanza-aprendizaje, fomenta la comunicación y el bienestar en la clase. (Sánchez, 2009, p. 154)

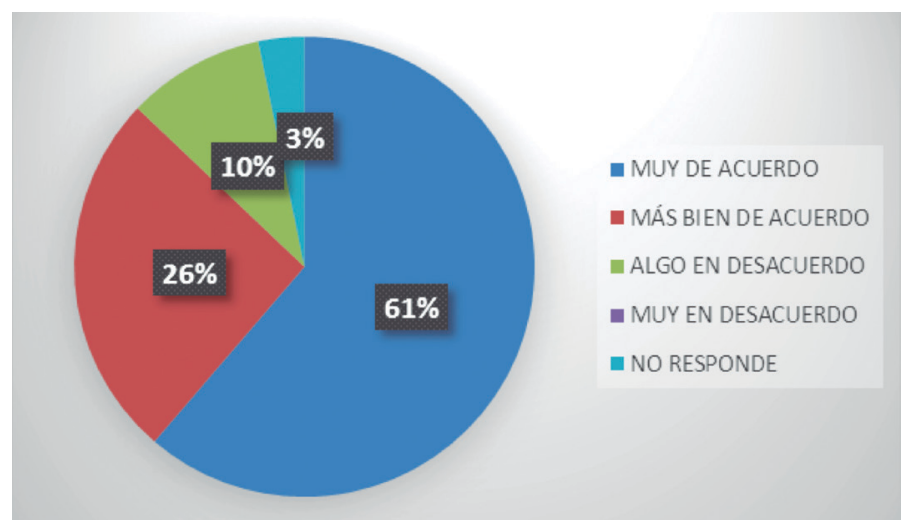

Fuente: encuesta aplicada a estudiantes de la Institución Educativa Técnica Internado San Francisco de Loretoyaco (Puerto Nariño, Amazonas).

Figura 2. Las normas de clase son claras para los estudiantes. 
Respecto a la claridad de las normas, el 61\% está muy de acuerdo en que las normas son claras. Un 26\% considera estar más bien de acuerdo, frente a un 10\% que refleja algo en desacuerdo.

Difícilmente se puede hablar de construcción participativa de normas cuando estas no son claras para los estudiantes, por tanto, es necesario evitar la ambigüedad de las mismas en la cotidianidad de las aulas. La claridad de las normas permite reflexionar sobre la importancia de aprender a vivir junto con los alumnos, fomentando espacios para la escucha, el diálogo, la sonrisa, conciliación y expresión de sentimientos. Los estudiantes muestran estar muy de acuerdo en la claridad de las normas pese a la dinámica intercultural que se vivencia, convirtiéndose en un proceso de construcción compartida de identidad cultural. La norma contribuye a la regulación de las relaciones de los estudiantes entre sí y con los demás integrantes de la comunidad educativa, por ello es conveniente comprender su naturaleza y su dinámica propia dentro del clima de aula para que los estudiantes le encuentren sentido a lo que hacen.

Acá cobra preponderancia el manual de convivencia escolar, ya que es el documento institucional donde, por lo general, se encuentran las normas que se socializan en el contexto de aula. Es una norma jurídica esencial porque tiene un carácter pedagógico e interviene el acto de educar.

De acuerdo con lo dispuesto en la Constitución Política de Colombia y en los artículos 73 y 87 de la Ley 115 de 1994, para favorecer la construcción de la democracia participativa todas las comunidades deben elaborar el manual de convivencia. (Peinado y Rodríguez, 2009, p. 140)

Por lo anterior, para la construcción, actualización, revisión y claridad continua del manual de convivencia, se hace necesario dar respuesta a las preguntas que se plantean en la guía 49 "Guías pedagógicas para la convivencia escolar" del Ministerio de Educación Nacional (2014):

¿De qué manera se construyeron los acuerdos que están incluidos en el manual de convivencia?

- ¿En qué forma va a participar la comunidad educativa en el proceso de actualización?

- ¿Cuáles son las situaciones que afectan la convivencia escolar que se deben incluir en el manual de convivencia?

- ¿Qué estrategias se van a utilizar para divulgar el manual de convivencia? ¿De qué manera lo planteado en el manual es pertinente y responde a la realidad de la Institución educativa? (p.32) 
Construcción de normas: una experiencia desde el clima de aula

\section{Participación en la elaboración de las normas}

En lo relacionado con la participación en la elaboración de las normas, se tienen presentes los siguientes dos indicadores: el profesor pide la opinión de los estudiantes acerca del funcionamiento de la clase y la participación de los estudiantes en la selección de las normas de clase (ver Figura 3).

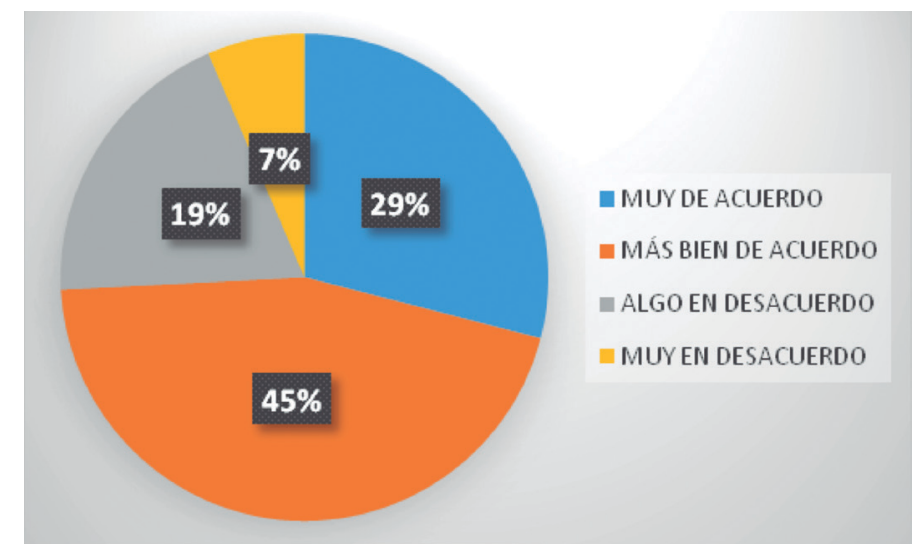

Fuente: Encuesta aplicada a estudiantes de la Institución Educativa Técnica Internado San Francisco de Loretoyaco (Puerto Nariño, Amazonas).

Figura 3. El profesor pide la opinión de los estudiantes sobre el funcionamiento de la clase.

En lo concerniente a la percepción de los estudiantes sobre el docente en el momento de dialogar respecto al funcionamiento de la clase, el 29\% de ellos considera estar muy de acuerdo en que estos espacios se generan dentro del aula. Un 45\% expresa estar más bien de acuerdo, frente a un 19\% que manifiesta estar algo en desacuerdo y el 7\% se encuentra muy en desacuerdo frente a la posibilidad de expresar sus percepciones y opiniones para el funcionamiento adecuado de las clases.

El tener en cuenta la opinión de los estudiantes puede convertirse en un hecho constructivo dentro del clima de aula, esto tiene que ver con no verlos como un código más, sino como seres humanos que tienen una vida compleja, una historia, unos intereses y una emocionalidad. Mirando y dialogando sobre las normas, se pueden tomar opciones de mejora y abordaje de los conflictos. Se evidencia que un alto porcentaje de los estudiantes muestran una percepción positiva frente a la posibilidad de ser agentes activos en este aspecto; sin embargo, es necesario continuar trabajando espacios de diálogo, ya que si no se tienen en cuenta las opiniones de los otros, difícilmente los conflictos se solucionarán; como lo expresa Aguilar (2011), 
"erradicar la cultura del silencio implica que los adultos asuman la responsabilidad que les cabe” (p. 43). Lo anterior, teniendo presente que la convivencia se construye en cada segundo que se pasa en el aula, incluido el proceso de enseñanza-aprendizaje, la misma no se construye solo a través de los equipos de mediación, el departamento de psicología o el comité de convivencia escolar.

La base de las relaciones humanas es la comunicación, pero ¿cómo desarrollar una comunicación efectiva si acallamos la voz del estudiante? Sin comunicación las cosas no funcionan. Para trabajar una buena comunicación dentro del clima de aula y fortalecer la construcción de normas de manera participativa, es necesario tener presente que debemos saber escuchar de manera activa y con apertura. Pero no solo es necesario saber escuchar, sino también saber hablar con claridad, respeto y valoración de la otra persona. A esta importancia de la palabra que es bella y armoniosa en la relación con el otro, Castañón (2013) la llama eufonía: "La eufonía sugiere una organización armónica, agradable y melodiosa de la palabra" (p. 290). Qué importante que pudiéramos trabajar la eufonía dentro de los climas de aula y la construcción de normas, porque, como lo expresa Castañón (2013) "la buena palabra empieza en la persona, en su mente, en su corazón, de donde brotan las palabras que ciertamente dicen mucho de si'” (p. 290).

Pero no solo es necesario tener presente la opinión del otro a través de la palabra dentro de la dimensión "claridad de la normas", sino también la forma como los estudiantes perciben su forma de participación dentro de la construcción de las mismas (ver Figura 4).

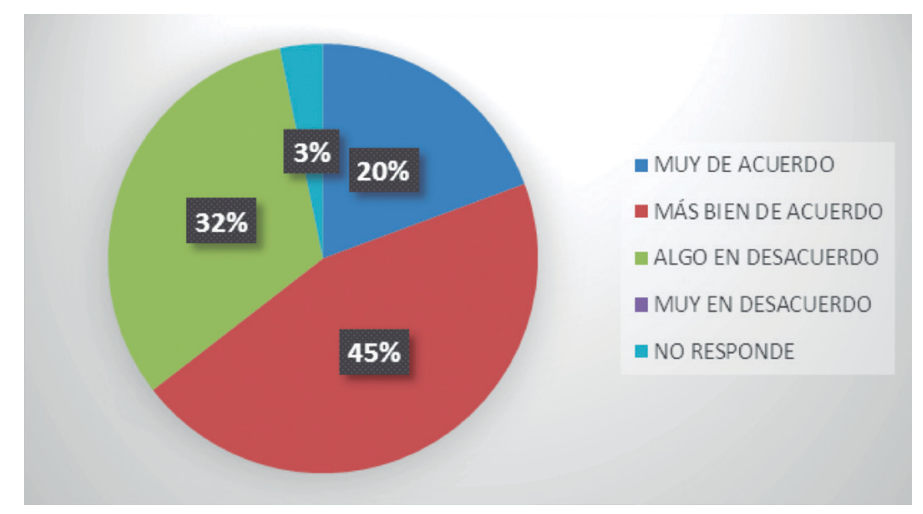

Fuente: Encuesta aplicada a estudiantes de la Institución Educativa Técnica Internado San Francisco de Loretoyaco (Puerto Nariño, Amazonas).

Figura 4. Los estudiantes participan en la selección de las normas de clase. 
Construcción de normas: una experiencia desde el clima de aula

Se puede constatar que el 45\% está más bien de acuerdo en que los estudiantes participan en la selección de la normas de clase, un 20\% considera estar muy de acuerdo, mientras que el $32 \%$ expresa estar algo en desacuerdo frente a la existencia de estos espacios dentro del clima de aula y un 3\% prefirió no responder.

A partir de las respuestas de los estudiantes se podría pensar sobre la necesidad de articular y vincular lo que aprenden los estudiantes de manera participativa con la formación ciudadana, con la realidad en la que viven. Intentar normativizar tanto de manera aislada e impuesta, impide que los estudiantes desarrollen sus ideas, como lo plantea Sánchez (2009), "la construcción de normas compartidas puede fortalecer sus vínculos y su identificación con el propio grupo” (p. 323). Los estudiantes deben entender que forman parte activa de un proceso formativo y, por lo tanto, pueden desarrollar el pensamiento propositivo de alternativas e implicarse en los procesos de toma de decisiones por medio del diálogo, la empatía y la escucha activa, como lo expresa Maturana (2007):

(...) todo lo que nosotros, los seres humanos hacemos como tales lo hacemos en conversaciones. Y aquello que no hacemos en conversaciones, de hecho no lo hacemos como seres humanos. En este proceso, los niños crecen como seres humanos entrelazando lenguaje y emocionalidad en su vida. (p. 46)

\section{Clima de aula}

Regular las vivencias dentro del clima de aula se convierte en uno de los principales retos de la cogestión entre docentes y estudiantes. Esta no se puede abordar desde una perspectiva individual, sino que requiere de una cierta consistencia mediante el trabajo en equipo, el cual debe romper determinados obstáculos como la falta de compromiso, conductas disruptivas y desmotivación. Al respecto Ausubel, Novak y Hanesian (1990) mencionan que "todo tipo de relación humana es mediada principalmente a través de variables motivacionales" (p. 4). Esto es necesario reflexionarlo ya que existe una relación directa entre las vivencias en el clima de aula y el clima escolar en general, como lo muestra Rodríguez (2015) al mencionar el impacto de los climas de escolares en las Instituciones Educativas (Figura 5): 


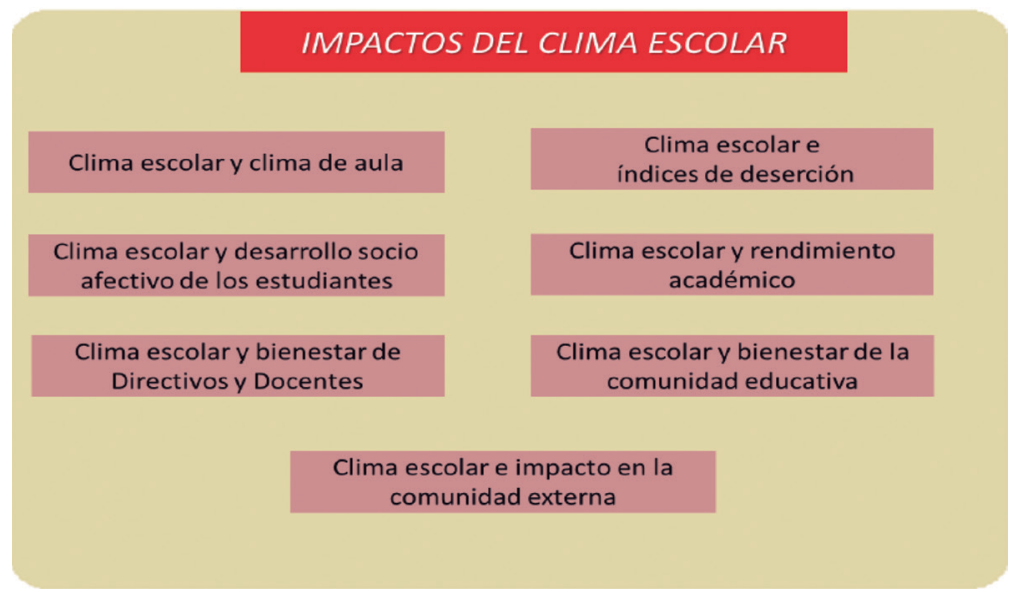

Fuente: (Rodríguez, 2015, p. 25).

Figura 5. Impactos del Clima escolar.

Por lo tanto, dentro de esta dimensión se aborda una categoría relacionada con el desarrollo de las clases a partir de dos preguntas específicas formuladas a los estudiantes, las cuales permiten identificar si en el curso toma algo de tiempo el inicio de las actividades programadas mientras se hace silencio, y la percepción de pérdida de tiempo en el desarrollo de las clases.

\section{Desarrollo de las actividades dentro del aula}

La concepción del centro escolar como un agregado de partes que tienen poco en común presenta limitaciones. Concebir la acción de educar a partir de expresiones relacionadas con percepciones aisladas como por ejemplo: ¡estos son tus estudiantes, no los míos; eso te corresponde a ti, no a mí!, hace que se asuma la educación bajo la orientación de seres aislados y no como un contingente donde todos somos responsables de la acción educativa.

Esta tendencia de responder solo por la parcela (aula) que me corresponde, genera que en ocasiones pervivan situaciones como la pérdida de tiempo al inicio de las clases y dentro del desarrollo de las mismas, lo que afecta los tiempos, metodologías y propósitos de formación inmersos en la planeación escolar. Lo anterior, lo podemos evidenciar en las respuestas de los estudiantes (ver Figura 6): 


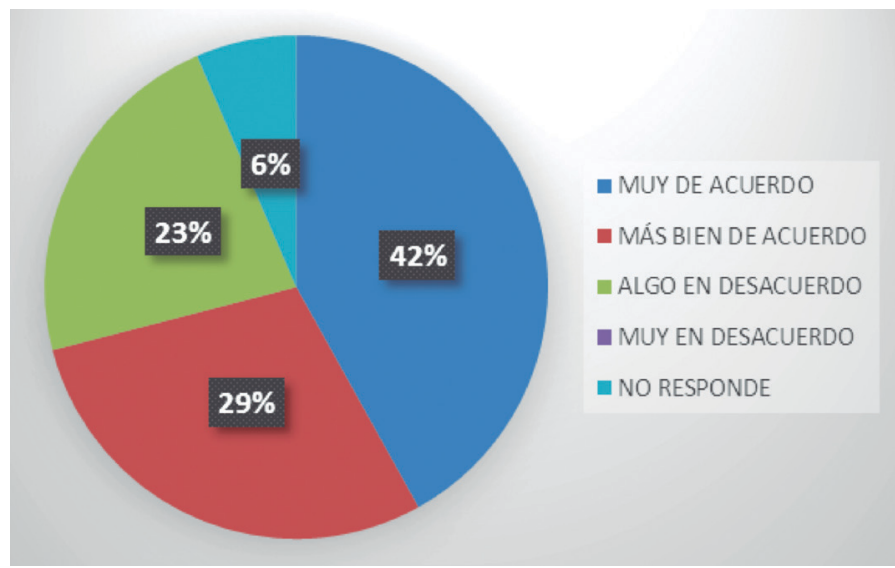

Fuente: Encuesta aplicada a estudiantes de la Institución Educativa Técnica Internado San Francisco de Loretoyaco (Puerto Nariño, Amazonas).

Figura 6. En el curso siempre toma algo de tiempo el inicio de las actividades planeadas.

La percepción sobre la pérdida de tiempo en el inicio de las actividades planeadas desde la visión de los estudiantes refleja que un 42\% de los estudiantes están muy de acuerdo en que se presenta esta situación de manera permanente, un 29\% muestra estar más bien de acuerdo, frente a un $23 \%$ que expresa estar algo en desacuerdo.

Uno de los temas que más se escucha en la cotidianidad de los contextos escolares es lo que sucede dentro de las aulas en la relación docente-estudiante o estudiante-estudiante. Acá podemos mencionar metodológicamente lo que aporta Baroccio (2013) como una de las ayudas prácticas sugeridas: “Aceptar, amar y alentar" (p.113). Como lo expresa la autora, “con una dosis muy baja de cualquiera de estos elementos, empezarán a ver una mejoría notable en sus alumnos que con sus comportamientos molestos están pidiendo ayuda a gritos" (Baroccio, 2013, p. 113). Analizar en el clima de aula los procesos de comunicación, las emociones, la toma de perspectiva y la empatía, es una responsabilidad de todos, los estudiantes son de todos.

Aprender a convivir con los demás es uno de los principios de la educación, en donde la escuela como un ecosistema social complejo en el cual cada individuo recibe y proporciona influencias en varios sistemas grupales interconectados y desde su especificidad, aporta importantes matices en todo lo concerniente a la intencionalidad preventiva y la eficacia interventiva para evitar las pérdidas de tiempo dentro del desarrollo de las clases.

Siempre se trata de sistemas de relaciones interpersonales, pero estas están siempre connotadas y en gran medida signadas por las tareas que los individuos deben desplegar en el interior de las 
mismas, por la actividad que une sus comportamientos en unos escenarios y en unos tiempos determinados (Ortega, 2005).

Otro aspecto relacionado con el desarrollo de las clases y que influye en las vivencias percibidas dentro del clima de aula, es no solo la pérdida del tiempo al inicio de las actividades, sino también en el desarrollo de las mismas (ver Figura 7). Potencializar de manera permanente habilidades y competencias sobre el manejo asertivo de las emociones y la toma de perspectiva frente a las situaciones que se presentan en la interacción diaria, se convierten en asuntos impostergables en el momento de generar conciencia con el compromiso que se tiene frente a los problemas y necesidades del otro.

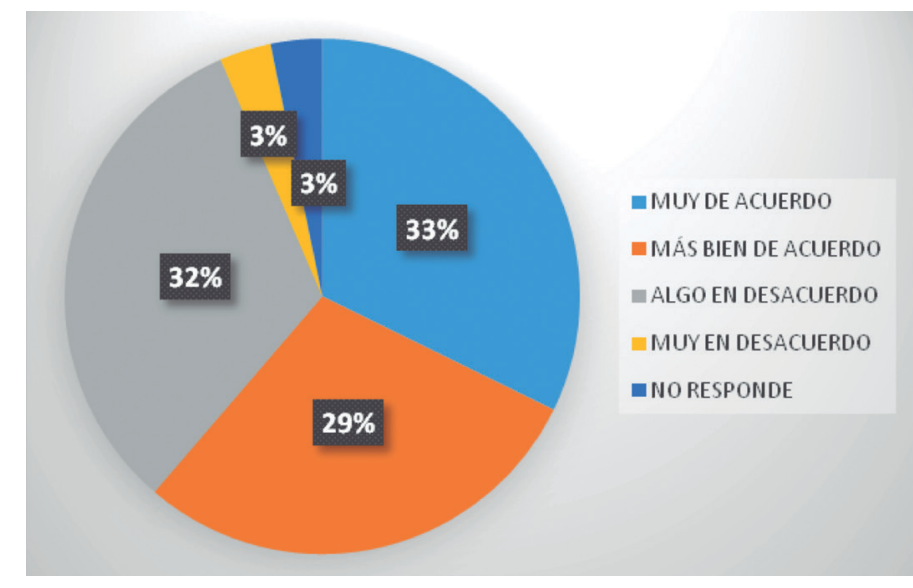

Fuente: Encuesta aplicada a estudiantes de la Institución Educativa Técnica Internado San Francisco de Loretoyaco (Puerto Nariño, Amazonas).

Figura 7. Se pierde mucho tiempo porque los estudiantes interrumpen la clase (hablan en voz alta, juegan, entre otros).

La figura anterior muestra que el 33\% de los estudiantes expresa estar más bien de acuerdo con que se pierde mucho tiempo en el desarrollo de las clases porque los compañeros tienen la tendencia a interrumpir el desarrollo de las mismas dentro del aula, un 29\% considera estar muy de acuerdo con la persistencia de esta situación. Asimismo, el 32\% manifiesta estar algo en desacuerdo y el 3\% muy en desacuerdo.

La mayoría de las dificultades en el aula suelen deberse a la falta de asertividad o respeto, también a la falta de empatía y autocontrol. Es necesario ubicar los problemas, esto significa que el problema finalice en el agente que tiene más probabilidades de resolverlo. No es conveniente que en educación abordemos los problemas desubicadamente. 
Construcción de normas: una experiencia desde el clima de aula

Las situaciones que afectan la sana convivencia dentro del aula tienen que estar ubicadas para que el sistema sea eficiente y la eficacia sea óptima. Disponer de estrategias de autocontrol, asertividad, empatía y aplicarlas automáticamente con cierta personalización pero de manera automática, permite abordar los problemas a través de una disciplina positiva. Al respecto, menciona Martínez (2014):

(...) la disciplina positiva se define como un programa o conjunto de actividades soportados por aquellas actitudes de los educadores que se orientan a guiar al estudiante en la mejor consecución de los objetivos de su formación tanto académica como personal y social. Por tanto, es una dimensión positiva, que contrasta con la dimensión negativa y sancionadora de la disciplina tradicional. (p. 41)

La disciplina positiva permite la optimización del tiempo, ya que éste pasa inexorablemente, el aprovecharlo depende de cada uno, invertirlo o gastarlo. ¿Cuántos espacios familiares, noches en vela planeando, pensamientos creativos y estrategias didácticas pueden perderse dentro del desarrollo de las clases por las interrupciones permanentes? Pues bien, es en el clima de aula donde podemos generar competencias ciudadanas, y no se trata de una tarea de vencer alumnos sino de convencerlos. A la hora de convencer y cambiar, es importante el poder interpersonal, el ejemplo y la influencia de quien aplica las estrategias. Como lo expresa Arévalo (2002):

(...) ciertas cualidades del profesor, como paciencia, dedicación, voluntad de ayudar y actitud democrática, favorecen el clima psicológico positivo en el aula; al contrario el autoritarismo, la enemistad y el desinterés pueden llevar a que exista un clima negativo en el aula. (p. 20)

\section{Conclusiones}

La vida misma, y por ende las situaciones que se presentan en el clima de aula siempre tienen soluciones. No nos estamos estrellando ante una pared porque por algún lado podemos encontrar posibilidades basadas en la confianza en nosotros mismos y en el otro con mucha gestión y fuerza interior. Se evidencia que existe una relación directa entre la construcción de normas y el clima del aula. Puede observarse en la construcción de normas que existe una claridad, ya que al comienzo del año escolar el docente explica la importancia de la buena conducta, asimismo los estudiantes perciben que son claras. El proceso permite constatar que la claridad y la socialización de la normas son aspectos necesarios para evitar ambigüedades en los espacios de fortalecimiento de las competencias emocionales, sociales y los procesos de enseñanza-aprendizaje. Somos productores de condiciones, podemos tomar el control de las situaciones y emprender la creatividad para las soluciones a los problemas. Esto tiene que ver con la proactividad del profesorado. 
Asimismo, el clima de aula está relacionado intrínsecamente con el grado de implicación que tiene el docente con los estudiantes y el grupo en general, de igual modo influye la asertividad para mantener una cultura democrática y participativa por medio del manejo de las emociones y el desarrollo de habilidades sociales que fortalezcan el valor del tiempo en el aula a través de la toma de decisiones responsables, empáticas y éticas. Igualmente, es necesario recordar el poder de la eufonía en el aula. Es importante enseñar a enfrentar de forma ética y eficaz los conflictos. Tenemos que aprender que disentimos, pero a través de un saber hablar, escuchar y por medio de una construcción de normas de manera participativa y la internalización de las mismas, de manera progresiva iremos favoreciendo los comportamientos prosociales, el éxito académico y el pasar de enseñar no solo lo que sabemos sino también lo que los estudiantes necesitan.

\section{Referencias}

Aguilar, M. (2011). La educación y la gestión de los conflictos. Montevideo, Uruguay: Concepto.

Arévalo, E. (2002). Clima escolar y niveles de interacción social, en estudiantes secundarios del colegio Claretiano de Trujillo 2002 (tesis de maestría). UNMSM, Lima, Perú.

Ausubel, D., Novak, J. y Hanesian, H. (1990). Psicología educativa: un punto de vista cognoscitivo. Ciudad de México, México: Trillas.

Baroccio, R. (2013). Disciplina con amor en el aula. Ciudad de México, México: Editorial Pax.

Besalú, X. (2002). Diversidad cultural y educación. Madrid, España: Síntesis.

Castañón, R. (2013). Cuando la palabra sana. La Paz, Bolivia: Grupo Internacional para la Paz.

Gómez, M., Mir, V. y Serrat, M. (2007). Propuestas de intervención en el aula. Técnicas para lograr un clima favorable en la clase. Madrid, España: Narcea.

Lipman, M. (1992). Filosofía en el aula. Madrid, España: Ediciones de La Torre.

Martínez, J. (2014). El manual de convivencia y la prevención del bullying. Bogotá, Colombia: Editorial Magisterio.

Martínez, M. (1996). La orientación del clima de aula. Investigación sobre el desarrollo de una investigación (tesis doctoral). Universidad Autónoma, Barcelona, España.

Maturana, H. (2007). Transformación en la convivencia. Santiago de Chile, Chile: JC Sáez Editor.

Ministerio de Educación Nacional. (2014). Guías pedagógicas para la convivencia escolar. Bogotá, Colombia: Ministerio de Educación Nacional.

Ortega, R. (2005). Estrategias educativas para la prevención de la violencia: mediación y diálogo. Madrid, España: Cruz Roja Juventud. 
Construcción de normas: una experiencia desde el clima de aula

Peinado, H. y Rodríguez, J. (2009). Manual de gestión y Administración Educativa: Cómo crear, gestionar, legalizar, liderar y administrar una Institución Educativa. Bogotá, Colombia: Editorial Magisterio.

Rodríguez, A. (2015). Clima Escolar. Una experiencia desde el municipio del Socorro - Santander. Tunja, Colombia: Editorial Fundación Universitaria Juan de Castellanos.

Sampieri, R. (1997). Metodología de la investigación. Ciudad de México, México: McGraw-Hill.

Sánchez, A, (2009). Acoso escolar y convivencia en las aulas. Manual de prevención e intervención. Granada, España: Formación Alcalá. 